\title{
A STRUCTURAL APPROACH TO THE HAZOP - HAZARD AND OPERABILITY TECHNIQUE
}

\author{
M. A. D. HERRERA ${ }^{1}$, A. S. LUNA ${ }^{2}$, A. C. A. da $\operatorname{COSTA}^{2}$, E. M. B. LEMES ${ }^{1}$ \\ ${ }^{1}$ Fundação Oswaldo Cruz/Bio-Manguinhos, Instituto Tecnológico de Imunobiológicos \\ ${ }^{2}$ Universidade do Estado do Rio de Janeiro, Instituto de Química, Programa de Pós- \\ graduação em Engenharia Química \\ e-mail para contacto: asluna@uerj.br
}

\begin{abstract}
Recently companies and corporations are increasing the use of analytical techniques to identify, assess and address risks, starting from the early stages of a project until the operation and production stage. This work is done with the intent to avoid deviations or minimize the impact if such deviations could occur in systems, processes, equipment and facilities. The hazard and operability study - HAZOP is a technique for identifying inductive risks based on the premise that accidents occur as a result of the deviation of process variables in contrast of the standard operating parameters. In this work, it is suggested the general methodology for applying the HAZOP technique within the industry through a structured process that can be followed easily by people who are interested in starting to use this technique to improve the security environment within the institution or when is required by regulatory agencies.
\end{abstract}

\section{INTRODUCCIÓN}

El análisis de riesgo ha sido utilizado por agentes financieros y de crédito por más de 70 años, así, la gestión de riesgo ha sido asociada en mayor parte con el mercado de seguros para proteger a los individuos y compañías de diferentes pérdidas asociadas con incidentes (Harrington and Neihaus, 2003). El estudio de operatividad y riesgos HAZOP, es una herramienta de análisis que fue desarrollada para identificar riesgos potenciales y problemas operacionales en plantas y procesos industriales que pueden comprometer tanto la productividad y seguridad de la planta, así como al proceso y producto. Esta técnica es utilizada como base para analizar cualitativamente los riesgos y problemas operacionales, principalmente durante la utilización de nuevas tecnologías o cuando es necesario incrementar el conocimiento sobre un nuevo proceso (Dunjó, 2010).

Esta técnica ha sido utilizad efectivamente en cualquier fase de la vida útil de plantas industriales dentro de la industria química, farmacéutica y de alimentos. Aunque es posible utilizar aplicar la herramienta de análisis de riesgo HAZOP en cualquier tipo de sistemas, en el caso particular de sistemas de alta complejidad esta implementación requiere del alto consumo de recursos tanto económicos como humanos sin contar el tiempo prolongado de elaboración de estudio. Por lo tanto los criterios de selección de cada uno de los elementos necesarios para la aplicación de la metodología serán los responsables para marcar la diferencia en la obtención de modelos reales durante la evaluación de riesgo. 


\section{OBJETIVO}

Proponer un procedimiento de aplicación de la herramienta de análisis de riesgo HAZOP y establecer los criterios de selección de nodos para la identificación, evaluación y tratamiento de los riesgos durante la calificación equipos, sistemas e infraestructura para atender los requisitos exigidos por agencias regulatorias

\section{METODOLOGÍA}

El procedimiento recomendado de aplicación de la herramienta HAZOP está representado en la figura 1 , donde preferentemente debe llevarse a cabo por un equipo de trabajo compuesto por especialistas de diferentes áreas tales como ingeniería, mantenimiento, automación, proceso, eléctrica y civil entre otros, bajo la supervisión de un líder que debe organizar las tareas y definir a los responsables por aplicar cada uno de los pasos de la metodología de una forma crítica, objetiva y antes que todo con enfoque técnico.

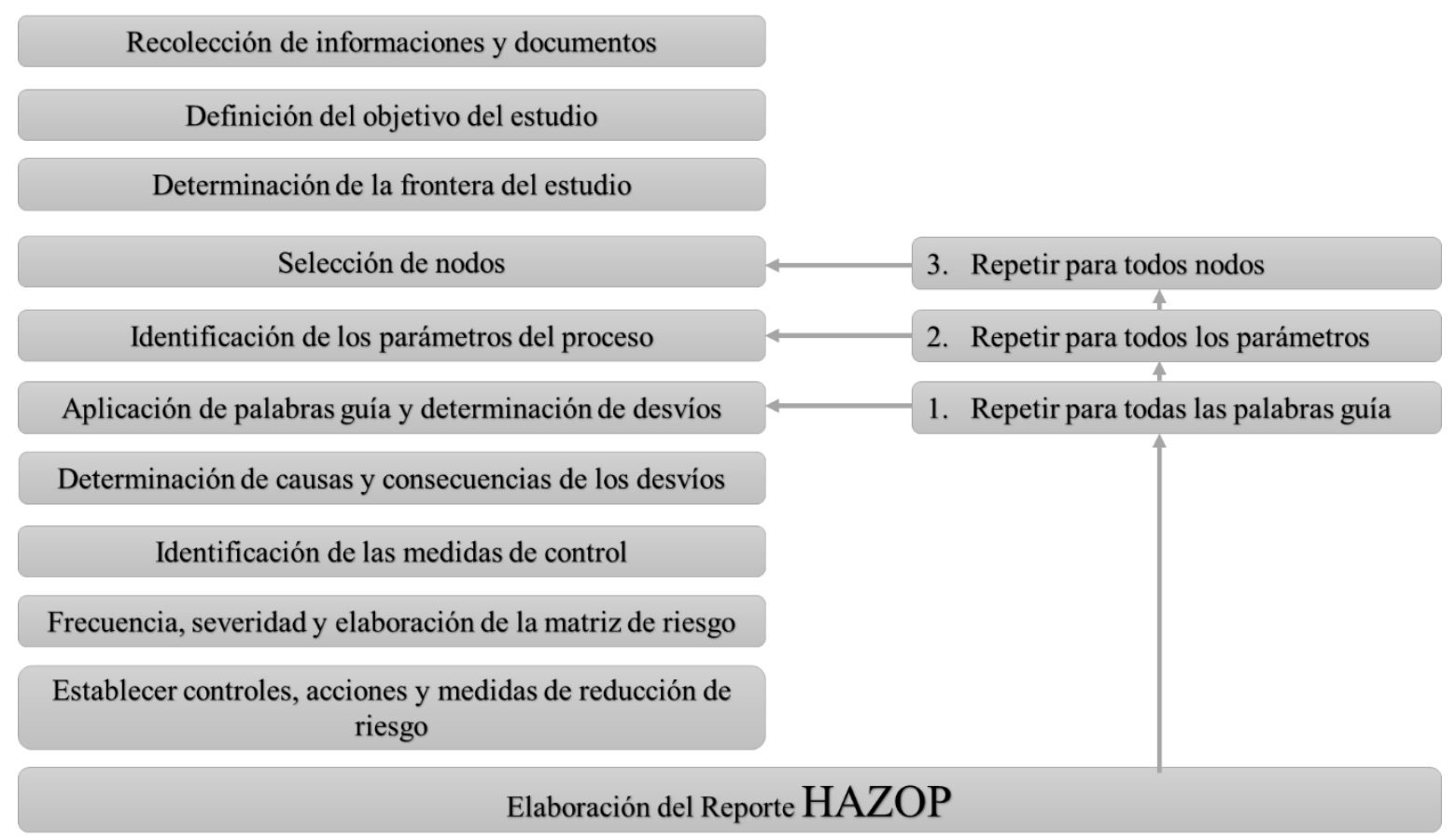

Figura 1 - Procedimiento de aplicación de la herramienta de análisis de riesgo HAZOP

Recolección de informaciones y documentos: Es la etapa inicial donde es recopilada toda la información técnica del sistema incluyendo diagramas de tuberías e instrumentación (PI\&D), diagramas de flujo de proceso, planos, manuales de equipos, etc.

Definición del objetivo del estudio: para llevar a cabo un análisis de riesgo es fundamental establecer la intención para aplicar la herramienta, este objetivo puede tener foco de seguridad industrial e infraestructura, ambiental, comercial, proceso, producto y clientes. Aunque es posible evaluar en diferentes áreas en paralelo, es recomendable establecer un único objetivo para evitar que criterios de análisis de peligros sean generalizados y pierdan validez en el sistema. 
Definición del límite de estudio del sistema/equipo/instalación: Es posible facilitar la aplicación de la herramienta a través de la reducción del área de análisis, permitiendo una evaluación fácil y objetiva.

Selección de nodos: Esta etapa es considerada como la más crítica, ya que únicamente con la identificación de los puntos críticos del sistema será posible la evaluación de los modos de falla de los sistemas.

Identificación de los parámetros de proceso: Es la caracterización de los elementos que constituyen los nodos, normalmente lo parámetros son parámetros físicos como temperatura, presión, flujo y volumen, aunque también son considerados químicos como concentración y reacción.

Aplicación de las palabras guía y desvíos: Las palabras guía son el modo de falla que existe en los parámetros de proceso, dando como resultado desvíos en el sistema. Estas palabras consisten en un proceso diferente o negativo en el peor de los casos a la función normal de la operación. Las palabras guía más comunes son Mas, Menos, Nada, etc. Que en conjunto con los parámetros resultan en desvios como por ejemplo, mayor flujo, menor flujo o sin flujo. Es importante recordar que este proceso se repite para todos los parámetros de cada nodo identificado.

Causas y consecuencias de desvíos: El equipo de especialistas tiene la tarea de establecer el origen y los efectos de los desvíos identificados, manteniendo en todo momento un fundamento técnico y real.

Identificación de las medidas de control: Las medidas de control son elementos que forman parte del sistema en estudio que tienen la función de reducir el impacto los desvíos.

El equipo de trabajo debe utilizar como fuente de información datos históricos y experiencia en otros procesos la frecuencia de que un evento pueda realizarse y el grado de impacto sobre el sistema. Con esa información es posible definir la matriz de riesgo que permitirá determinar el nivel de riesgo de nuestro sistema.

Utilizar una matriz de riesgo ofrece en cualquier situación soporte para evaluar de forma precisa el efecto resultante de un desvío, esto es realizado a través de la medición del impacto de un evento así como la frecuencia o probabilidad de que este se lleve a cabo. La dimensión de la matriz de riesgo se define a criterio particular del equipo de trabajo, tanto en los intervalos de frecuencia así como en los niveles de impacto de eventos, sin embargo se recomienda que el equipo de trabajo establezca escenarios reales de evaluación de los desvíos de operación del sistema.

En la tabla 1 puede ser observado un ejemplo de elaboración de la matriz de riesgo, considerando seis niveles de frecuencia cinco niveles de severidad o impacto de los desvíos. 
Tabla 1 - Matriz de evaluación de riesgo

\begin{tabular}{|c|c|c|c|c|c|c|c|}
\hline \multicolumn{8}{|c|}{ Matriz de Riesgo } \\
\hline \multicolumn{2}{|c|}{ Frecuencia } & Improbable & Remoto & Ocasional & Probable & Frecuente & Continuo \\
\hline \multicolumn{2}{|c|}{ Severidad } & 1 & 2 & 3 & 4 & 5 & 6 \\
\hline Insignificante & 1 & Insignificante & Insignificante & Insignificante & Aceptable & No deseable & асере \\
\hline Baja & 2 & Insignificante & Insignificante & Aceptable & No deseable & Inaceptable & Inaceptable \\
\hline Moderada & 3 & Insignificante & Aceptable & No deseable & No deseable & Inaceptable & Inaceptable \\
\hline Crítica & 4 & Insignificante & Aceptable & No deseable & Inaceptable & Inaceptable & Inaceptable \\
\hline Catastrófica & 5 & Aceptable & No deseable & Inaceptable & Inaceptable & Inaceptable & Inaceptable \\
\hline
\end{tabular}

\section{RESULTADOS}

La herramienta de análisis de riesgo HAZOP fue aplicada en la determinación los puntos críticos que deben ser monitoreados para la calificación (validación de equipos) de un sistema de biorreacción de células procariotas mostrado en la figura 2 constituido por dos fermentadores, dos tanques de servicio y la tubería de conexión de sistema que será utilizado en la producción de proteínas recombinantes para insumos farmacéuticos.

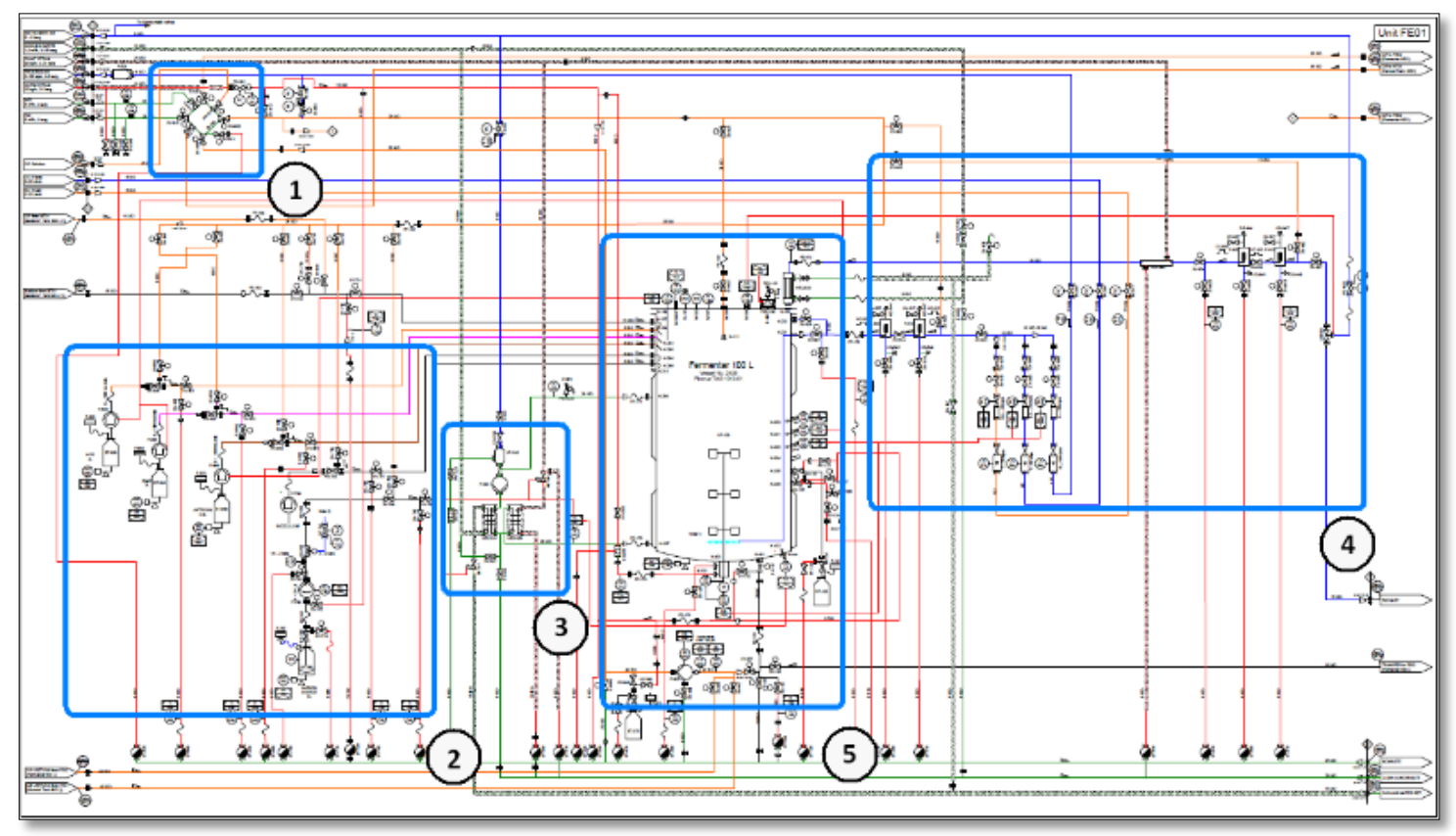

Figura 2 - Sistema de biorreacción con cinco puntos críticos identificados

Cinco nodos fueron identificaron en el sistema biorreacción. El primer nodo identificado como el sistema de distribución de agua de inyección, agua purificada, vapor puro y la solución desinfectante fue caracterizado con niveles de riesgo en mayor parte aceptables. El segundo nodo de las líneas de transferencia de soluciones de biorreacción mostró niveles de riesgo aceptables. El tercer nodo sistema de intercambiadores de calor de la misma forma tuvo niveles aceptables de riesgo así como niveles indeseable. El cuarto nodo que se caracterizó como la filtración de gases de proceso y sistema de escape mostró un nivel aceptable de riesgo. El quinto nodo identificado como el tanque principal obtuvo niveles insignificantes y aceptables de riesgo. 


\section{CONCLUSIONES}

La metodología HAZOP es una poderosa herramienta para la identificación, evaluación y reducción de riesgos en los procesos continuos, por lotes o semi-continuos.

La aplicación del procedimiento propuesto de esta herramienta de análisis de riesgos en el sistema estudiado permitió identificar sus puntos críticos, reduciendo el tiempo y facilitando el proceso de calificación de ese sistema cumpliendo asi con los requisitos exigidos por la agencia regulatoria.

Fue comprobado que agrupar diferentes componentes con funciones similares así como aquellos con consecuencias y efectos equivalentes para definir nodos, redujo ampliamente el tiempo de aplicación de la herramienta sin comprometer la profundad y eficiencia del estudio.

\section{BIBLIOGRAFÍA}

DUNJÓ D. J. New trends for conducting hazard \& operability (HAZOP) studies in continuous chemical processes. Centre d'Estudis del Risc Tecnologic - 119 CERTEC. Departament d'Enginyeria Química - DEQ Escola Tècnica Superior d'Enginyers Industrials de Barcelona - ETSEIB - Universitat Politècnica de Catalunya - UPC, 2010.

HARRINGTON, S., NIEHAUS, G.R., Risk Management and Insurance, USA, Irwin/McGraw-Hill, 2003. 